\title{
IN VIVO RED CELL GLYCOLYTIC CONTROL AND DPG-ATP LEVELS *
}

\author{
G. J. Brewer, F. J. Oelshlegel, Jr., L. G. Moore, and N. A. Noble \\ Department of Human Genetics \\ University of Michigan Medical School \\ Ann Arbor, Michigan 48104
}

\section{INTRODUCTION}

Much of clinical medicine involves a struggle against hypoxic diseases (such as heart disease, pulmonary disease, and anemia), all of which owe their morbidity and mortality ultimately to inadequate tissue oxygenation. Because of the quantitative significance of these kinds of diseases in health today, it is particularly important to try to understand and exploit the possible compensatory mechanisms for inadequate tissue oxygenation.

FIgURE 1 puts the role of the red cell in perspective with other factors influencing tissue oxygenation. As the figure indicates, the factors that influence tissue oxygenation can be considered under four categories: cardiac output, pulmonary oxygen exchange, red cell oxygen transport, and lastly, hematological parameters, that is, red cell mass and amount of hemoglobin per red cell. Of course, an abnormality in any one of the four oxygen transport factors can be compensated somewhat by alterations in the other three factors. For instance, anemia leads to an increase in cardiac output and hyperventilation as well as to changes in red cell oxygen transport. Our emphasis in this paper, however, centers on those changes within the individual red cell that may improve its oxygen delivery during each transit through the tissue. Potentially, the most important change in this regard is a decrease in the oxygen affinity of the red cell (a shift to the right of the oxygen dissociation curve).

A question of importance that has not yet been answered is whether or not oxygen affinity within the ranges seen normally and pathologically in various mammalian species really influences function. Theoretically, it can be shown in man that a $3 \mathrm{~mm}$ right shift in the oxygen dissociation curve, as might be produced by a $25 \%$ increase in DPG, $\dagger$ has a potential for $22 \%$ greater oxygen transport if cardiac output, ventilation, and hemoglobin levels remain the same. ${ }^{1}$ This theoretical benefit is based upon essentially a resting situation in which blood with a normal oxygen affinity returns to the lungs with approximately $70 \%$ of its oxygen remaining, whereas with a decrease in oxygen affinity the blood returns more deoxygenated. With stress such as exercise or in certain organs such as the heart, desaturation is more complete and the theoretical benefit of decreased oxygen affinity is relatively less. The effect of changes in oxygen affinity can also be examined in terms of the hypothetical drop in tissue

* This work was supported by the Department of the Army and Navy under Contract DADA17-69-C-9103, National Institutes of Health Training Grant 5T01-GM00071-14, The Michigan Heart Association, and a grant from the A. H. Meyers Foundation.

$\dagger$ See legend to Figure 2 for key to abbreviations. 


\section{GENES AND/OR ENVIRONMENT MAY INFLUENCE ALL OF THESE FACTORS}
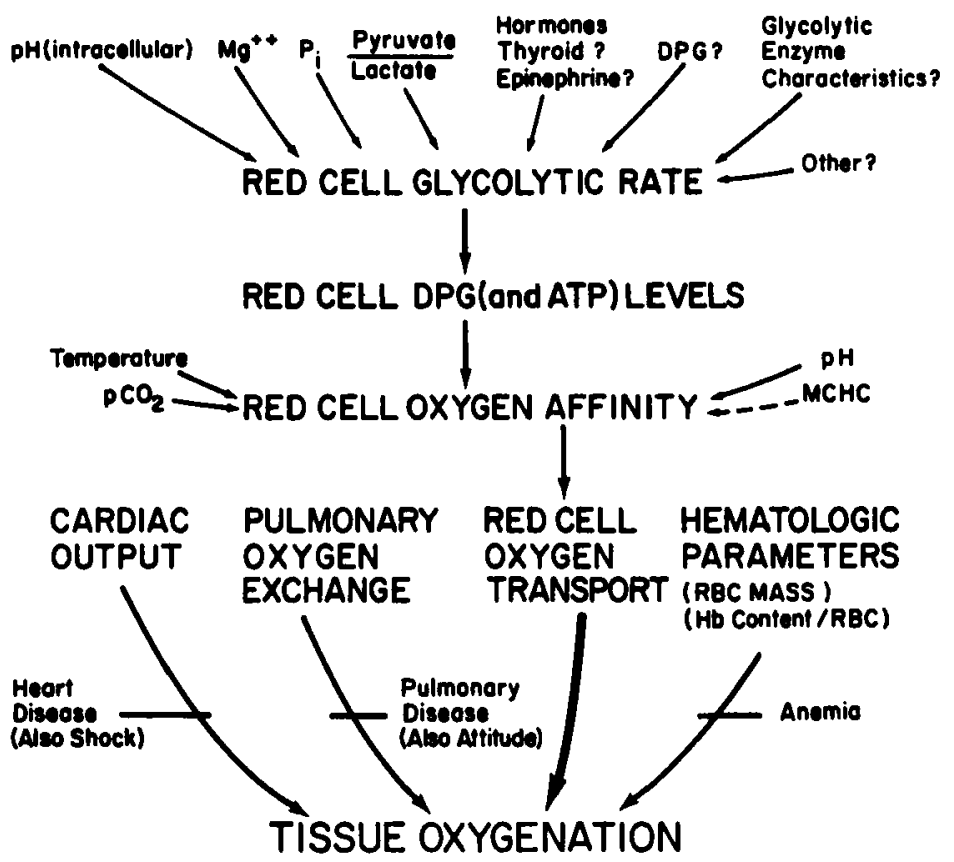

FIGURE 1. Schematic organization of factors influencing tissue oxygenation. Reprinted by permission of Academic Press.

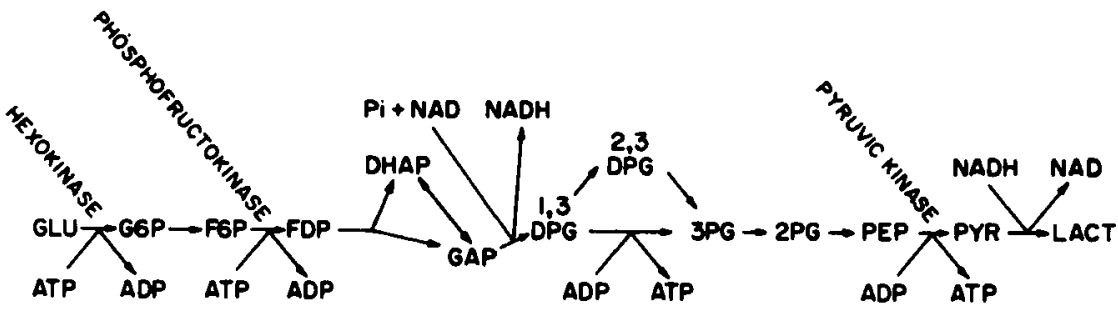

Figure 2. The glycolytic pathway of red cell metabolism. The three potentially most rate-limiting enzymes are identified. Abbreviations: GLU, glucose; ATP and $A D P$, adenosine triphosphate and diphosphate, respectively; G6P, glucose 6-phosphate; F6P, fructose 6-phosphate; FDP, fructose, 1, 6-diphosphate; DHAP, dihydroxyacetone phosphate; GAP, glyceraldehyde-3-phosphate; $P_{1}$, inorganic phosphate; NAD and $\mathrm{NADH}$, oxidized and reduced nicotinamide adenine dinucleotide, respectively; 1,3 DPG, 1,3-diphosphoglycerate; 2,3 DPG, 2,3-diphosphoglycerate; 3PG, 3-phosphoglycerate; 2PG, 2-phosphoglycerate; PEP phosphoenol pyruvate; PYR, pyruvate; LACT, lactate. 
capillary oxygen tension that must be sustained in order for red cells with relatively greater affinity to deliver the amount of oxygen equivalent to that of red cells with decreased affinity. Theoretically, the difference in tissue capillary oxygen tension in $\mathrm{mm} \mathrm{Hg}$ is approximately the same as the difference in oxygen affinity. Whether oxygen tension differences are critical to tissues is not known. The concept of a "critical tissue oxygen tension" is relatively unexplored.

Throughout this paper we will make the tacit assumption that variation in oxygen affinity does have important effects on oxygen transport and on at least some aspects of function of the organism. In view of the above discussion, it should be kept in mind that this remains to be proven because, with the exception of skeletal muscle performance, the data are not available. If we assume that red blood cell oxygen affinity is important in oxygen transport, then an understanding of the regulation of DPG in the red cell becomes important. Of the various factors that influence oxygen affinity in the intact red cell, only DPG shows sufficient variability to influence significantly red cell oxygen affinity. Further, since DPG is intimately associated with red cell glycolysis, in order to understand red cell DPG variability we must understand red cell glycolytic control. Equally important to DPG is the level of red cell ATP, and its regulation.

\section{Control of Red Cell Glycolysis}

FIGURE 2 shows the intermediates of the glycolytic pathway with the three probable most rate-limiting enzymes in the human red cell identified. These are: hexokinase (HK), phosphofructokinase (PFK), and pyruvate kinase (PK). The strongest evidence for the rate-limiting nature of these enzymes under normal conditions are the calculations of Minakami and Yoshikawa, ${ }^{2}$ who used the free energy changes of various steps in glycolysis to identify those enzymatic reactions that are far removed from thermodynamic equilibrium (FIgURE 3). This study clearly identified the HK, PFK, and PK reactions as far removed from equilibrium, making these three enzymatic reactions likely controlling steps for various portions of the glycolytic pathway. In addition to the work of Minakami's group, Rapoport and his group ${ }^{3+}+$ have contributed extensively to an understanding of red cell glycolytic control. Both the Minakami and Rapoport groups (best summarized in the published discussion after Yoshikawa and Minakami's 1968 paper ${ }^{5}$ ) believe that red cell glycolytic control is not exercised by one enzyme, but is the result of a number of positive and negative factors operating on at least these three enzymes.

While in this paper we do not have space to expand extensively upon the previous work suggesting a rate-limiting nature for each of these three enzymes, one factor for each enzyme may be worthy of comment. First, HK is unusual among the glycolytic enzymes in that its capacity (Vmax) is not much beyond the glycolytic rate of intact red cells, at least in the human. This alone suggests an important rate-limiting function for this enzyme. For PFK very good evidence exists that indicates that the enzyme is extremely sensitive to $\mathrm{pH}$, being activated by an increase in $\mathrm{pH}$. It has been shown that the glycolytic intermediates prior to the PFK step decrease, and the glycolytic products subsequent to the PFK step increase, in association with an increase in glycolytic rate upon an increase in $\mathrm{pH}^{8}{ }^{8}$ This indicates an important regulating role for PFK, particularly in response to $\mathrm{pH}$ variation. Finally, interesting observations exist 


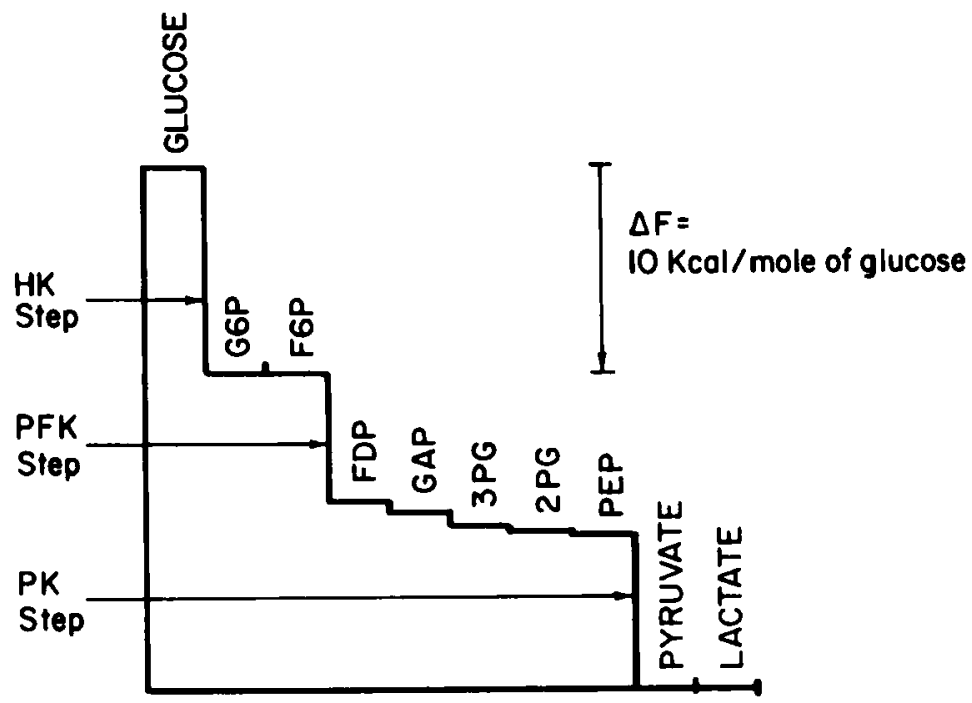

Figure 3. Free energy changes at various steps in the glycolytic pathway as calculated by Minakami and Yoshikawa. ${ }^{2}$ Note the large changes at the HK, PFK, and PK steps. Reprinted by permission of the Japanese Biochemical Society.

with respect to $\mathrm{PK}$ activity, not so much as influencing overall glycolytic rate, but in terms of an effect upon the relative amounts of DPG and ATP. It is well established that in PK deficiency the level of DPG is relatively high compared to that of ATP. On the other hand, a family reported by Zürcher and colleagues ${ }^{7}$ showed inherited variation in which the maximal activity of PK was high, and the red cells had relatively low levels of DPG and high levels of ATP. These observations suggest to us that the ratio of DPG to ATP, at least in the human, depends upon PK activity.

\section{USE OF INTERMEDIATE ASSAYS FOR In Vivo SITUATIONS}

The previous section introduced the concept of the usefulness of studies of intermediates in the glycolytic pathway in understanding the dynamics of control of that pathway. Most of the studies mentioned above have involved measurement of glycolytic intermediates either in normal blood or during in vitro incubations. It is equally possible, however, to learn a great deal about glycolysis in various in vivo situations, such as stress or disease, by studies of glycolytic intermediates in the red cell in these conditions. In this section we will briefly discuss the use of this approach in a number of situations.

\section{Elevated Erythrocyte ATP}

In 1965 , a family segregating for a gene determining very high levels of red cell ATP was described. ${ }^{8}$ This gene is inherited as an autosomal dominant, with 
some variability in expression. At the time of the original study no primary biochemical defect was discovered. This family has recently been restudied using the approach of assay of glycolytic intermediates. ${ }^{9}$ The findings are illustrated in FIgURE 4. It was observed that in addition to elevated levels of ATP, the levels of four intermediates, 2,3-DPG, 3-PG, and PEP, were all significantly reduced. In preliminary studies in one affected person, red cell glucose consumption was normal. The interpretation that seemed most likely to us was an increase in the in vivo activity of PK that might-in the manner of the family described by Zürcher and colleagues ${ }^{7}$-increase ATP at the expense of DPG. However, the Vmax for PK was actually lower than normal in affected members of this family. ${ }^{9}$ The probable explanation, however, was found when the kinetics of the PK enzyme were studied. It was found that the $\mathrm{Km}_{\mathrm{PEP}}$ for PK was abnormally low in affected members. ${ }^{9}$ This indicates that the in vivo functioning of the enzyme under conditions of PEP limitation (as exists in vivo), is greater than that of the normal enzyme.

The main point we wish to make from the study in this family is that the use of intermediate assays is extremely valuable in the study of cellular metabolic abnormalities. Enzyme activity ( Vmax) alone is inadequate. A second point bears more specifically upon the role of $P K$ in regulating the ratio of DPG to ATP in the red cell. The ratio of DPG to ATP has very important implications because these two substances have very different functions. The activity of PK appears to play an important role in determining the level of ATP vs DPG.

\section{Hypoxia}

\section{Anemia}

Assay of glycolytic intermediates has also been very useful in the study of red cell adaptation to hypoxia, such as anemia. Glucose consumption has been shown to be markedly elevated in anemia. ${ }^{10}$ FIGURE 5 shows average intermediate values of 19 anemic subjects compared to control values.11 These subjects include both hemolytic and nonhemolytic anemias in about equal proportion. The qualitative patterns were quite similar irrespective of the etiology of the anemia, with an uniform increase in levels of all intermediates beginning with G6P. These patterns are interpreted as indicating $\mathrm{HK}$ activation. In

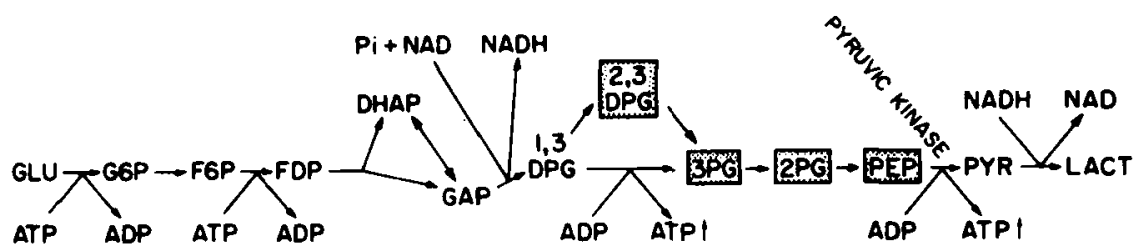

Figure 4. The glycolytic pathway illustrating the intermediate changes noted in individuals with inherited elevated erythrocyte ATP. The elevated ATP is symbolized by the arrows pointing up, and the intermediates with significantly reduced levels are enclosed in shaded boxes. The PK in these individuals has a reduced Vmax but also has a low Kmpep. 


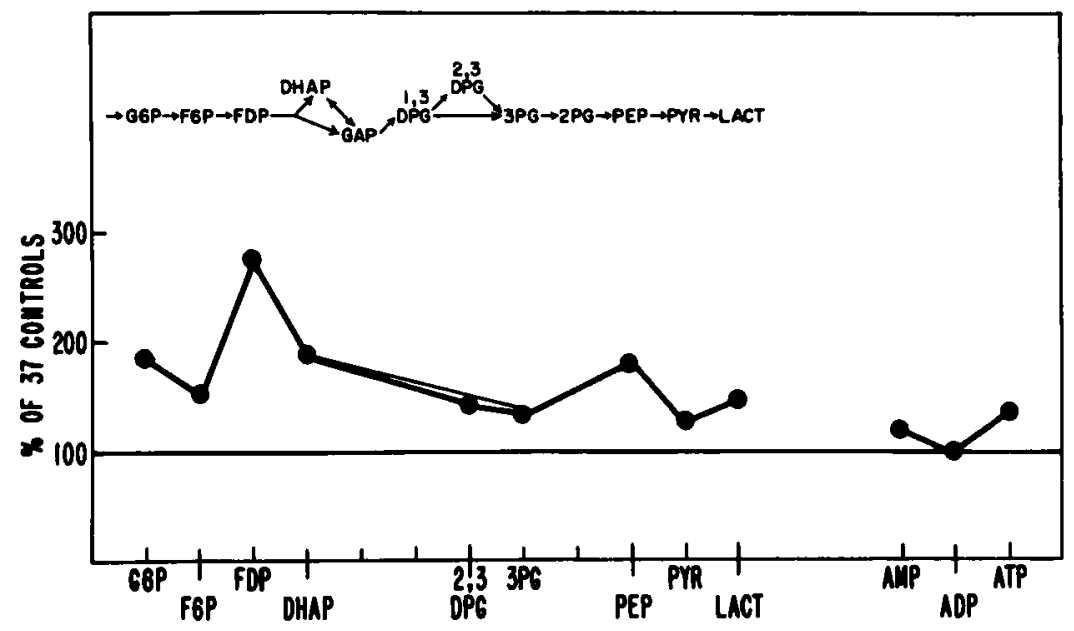

FiguRE 5. Average intermediate values of 19 anemics compared to control. Reprinted by permission of Plenum Press.

addition, in about half of the cases, the level of FDP is elevated out of proportion to the other intermediates, suggesting an additional PFK activation in these cases. The mechanism of the PFK activation appears to be hyperventilation alkalosis. ${ }^{11}$ The mechanism of HK activation is unknown.

\section{Altitude-Qualitative Observations}

Another type of hypoxic stress in which studies of red cell glycolytic intermediates has been useful is acute altitude adaptation. During studies of acute exposure to altitude of either $11,200 \mathrm{ft}$. or $14,100 \mathrm{ft}$., individual patterns suggestive of HK or PFK activation are frequently seen.12 There is, however, considerable variation in pattern from individual to individual and at different times in the first few days of exposure. A mean increase in G6P occurs that precedes the increase in DPG, and this may be interpreted as a qualitative indication that HK activation is generally important in adapting to acute altitude exposure. The qualitative interpretation of these patterns is difficult, however. Also, unlike the case with anemia it has not been possible, at least in our hands, to consistently show an increase in glucose consumption during altitude exposure. However, glucose consumption is measured in vitro. It is quite possible that the stimuli that act in altitude exposure to increase glycolysis require that the red cell be present in the whole organism.

\section{Altitude-Red Cell Metabolism as a System}

It should be clear from the foregoing discussions that red cell glycolysis is reasonably complex, with at least three enzymes, HK, PFK, and PK involved as rate-limiting in one manner or another, and many potential positive and 
negative effectors of each. It is difficult by the use of simple qualitative descriptive methods, such as glycolytic plots, bivariate correlation, and so forth, to understand and describe how red cell glycolysis is regulated over time during acute stress because of the complexity of the system and the number of variables involved. For this reason, we have turned to more powerful analytical approaches. ${ }^{13}, 14$ We will describe some of these briefly to illustrate the potential strengths of these approaches.

TABLE 1 lists the variables measured in two of our recent studies of altitude adaptation in human subjects. These variables were each measured on six occasions during each study. More than half of them changed significantly at altitude. A very important and practical problem arises, particularly in a small sample of subjects in which the degrees of freedom are limited, as to how to deal with so many variables. Obviously a sorting process of identifying "key" variables ("key" in the sense of being most important to DPG regulation) would be extremely useful.

Such an approach has been attempted with some preliminary success. ${ }^{13}, 14$ First, the linear dependence of DPG on each variable, one at a time, was determined for each of the two data sets (the two field trips). Then, an arbitrary screening procedure was devised by which the number of statistically significant entries determined inclusion of that variable as a key variable. This procedure identified G6P, PEP, PL $\mathrm{Mg}^{++}, \mathrm{RBC} \mathrm{Mg}^{++}$, FDP, and ATP as key variables in contributing to DPG change at altitude in the two field studies. An additional variable, 3PG, was added to this list on less rigorous grounds.

Some independent support for the importance of these variables exists. In anemia, G6P and FDP have already been mentioned as involved in DPG buildup. In addition, $\mathrm{RBC} \mathrm{Mg}^{++}$appears to be related. ${ }^{15}$ The identification of three of the same variables by the screening method described in the previous

\section{TABLE 1}

Variables Measured in Two Recent Studies on Human Altitude Adaptation

\begin{tabular}{ll}
\hline Hb (hemoglobin) & PLMG (plasma magnesium) \\
HCT (hematocrit) & RBC NA (red blood cell sodium) \\
MCHC (mean cell hemoglobin concentration) & RBC K (red blood cell potassium) \\
WBPH (whole blood pH) & RBC CL (red blood cell chloride) \\
ICPH (intracellular pH) & RBC MG (red blood cell magnesium) \\
DPG (2,3-diphosphoglycerate) & DPG/L (DPG per liter rbc water) \\
ATP (adenosine triphosphate) & ATP/L (ATP per liter rbc water) \\
ADP (adenosine diphosphate) & ADP/L (ADP per liter rbc water) \\
AMP (adenosine monophosphate) & AMP/L (AMP per liter rbc water) \\
G6P (glucose-6-phosphate) & G6P/L (G6P per liter rbc water) \\
F6P (fructose-1,6 phosphate) & F6P/L (F6P per liter rbc water) \\
FDP (fructose diphosphate) & FDP/L (FDP per liter rbc water) \\
DHAP (dihydroxyacetone phosphate) & DHAP/L (DHAP per liter rbc water) \\
3PG (3-phosphoglycerate) & 3PG/L (3PG per liter rbc water) \\
2PG (2-phosphoglycerate) & 2PG/L (2PG per liter rbc water) \\
PEP (phosphoenol pyruvate) & PEP/L (PEP per liter rbc water) \\
PYR (pyruvate) & PLNA (plasma sodium) \\
LACT (lactate) & PLK (plasma potassium) \\
PHOS (plasma phosphorus) & PLCL (plasma chloride) \\
\hline
\end{tabular}


paragraph adds confidence to the approach. Even stronger support for the importance of the key variable set comes from independent studies of the most hypoxic chronic residents of intermediate altitude $(10,200 \mathrm{ft}$.). These are the people with excessive polycythemia. The DPG of these polycythemic individuals showed much stronger dependence on the key variable set when compared to other variable combinations. ${ }^{13.14}$ These observations add independent support that the process resulting in the selection of the key variables based on shortterm data accurately selected the variables most related to hypoxic stress.

Principal component techniques have served as one of the exploratory tools for characterizing the multivariate correlation structure of the red cell system during short term altitude adjustment. ${ }^{13,14}$ By this approach two kinds of information have been developed. First, a certain portion of the relationships among the key variables (including DPG) in our various altitude studies can be expressed along one continuum as a single, newly defined, variable. This averaged $40 \%$ during acute adjustments, with about a $5-10 \%$ increase during early exposure, suggesting a "tightening up" of variable relationships associated with DPG buildup. Further support for the significance of this approach comes from observations on altitude residents. The average percent of the relationships attributable to the first principal component was about $30 \%$ in normal altitude residents, and $54 \%$ in altitude polycythemics, and $27 \%$ in sea level normals. Differences among these values for each sample are viewed as reflecting the tightness or cohesiveness of relationships between variables.

The second kind of information obtainable from principal component analysis refers to the relative contributions of single variables or groups of variables to the first principal components. DPG appears as the only variable with consistently high ranking contributions, providing support for modeling DPG as the dependent variable in the red blood cell system. In other words, the principal component technique provides a posteriori evidence for considering DPG as having the central role during altitude adjustment.

This brief discussion of analytical techniques is not intended to be comprehensive. For one thing, the amount of data is limited. But as the data-base increases, we anticipate increasingly useful information from a strong analytical approach. In addition, these same approaches can be applied to the red cell metabolic data in a variety of circumstances.

\section{Genetic Studies of Rat DPG and ATP Levels}

Previous work has shown that the levels of red cell ATP are inherited in humans, probably in a multigenic manner. ${ }^{16}$ Studies of the inheritance of human red cell DPG levels have not been made, but we have carried out genetic selection studies of DPG in Hooded Rats. These studies have shown strong genetic contributions to the quantitative level of red cell DPG in this animal. ${ }^{17}$ Frgure 6 shows the result of selection on DPG in Hooded Rats over a period of eight generations. Two strains with nonoverlapping values of DPG have been obtained. The high DPG strain has an oxygen affinity which is $6 \mathrm{~mm}$ greater than that of the low DPG strain. Unlike the human, the rat has a strong positive correlation between DPG and ATP levels. Previous work, ${ }^{17}$ using the approach of glycolytic intermediates assay, has revealed marked differences in the in vivo PFK activity in the two strains. The high DPG strain shows patterns in which the G6P and F6P levels are low, and the intermediates 
subsequent to the PFK step are high. This indicates greater in vivo PFK activity in the high strain. So far the exact mechanism of these PFK differences have not been established. Studies of maximal activities and the kinetics of PFK have not revealed differences between the enzymes of the two strains. ${ }^{18}$ However, we have found minor differences in a number of factors that affect PFK activity, such as inorganic phosphate level and $\mathrm{pH}^{1{ }^{18}}$ Our working hypothesis is that in our selection on DPG levels, we have selected for a number of factors, many of which influence PFK activity. ${ }^{18}$

Preliminary studies by Noble ${ }^{18}$ have revealed a number of interesting facets of these rat lines that should be further explored. First, the rats of the two lines have significant behavioral differences. Second, there are fertility and

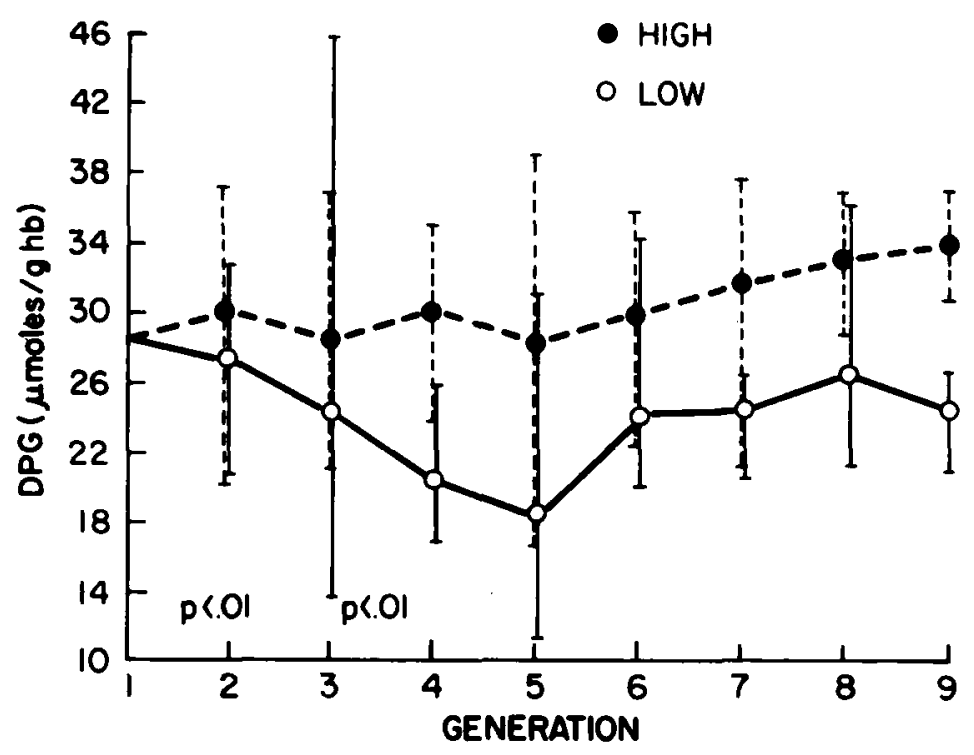

FIGURE 6. Separation of mean red cell DPG levels in Hooded Rats by generations of selective breeding. Two lines have been selected, designated "high" and "low". The mean and range of the offspring for each generation are shown.

possibly sex-ratio differences. Third, DPG of the $F_{1}$ of crosses between high and low rats is quite similar to the high strain, indicating some dominance of the gene(s) for the high trait.

\section{Pharmacological Manipulation of DPG levels and Oxygen Affinity}

Considerable interest has developed in the possibility of pharmacologically altering DPG levels in order to alter red blood cell oxygen affinity. Such work assumes, of course, that variability in oxygen affinity affects oxygen transport in a meaningful way, at least under some circumstances. Brain and Card ${ }^{19}$ used single doses of oral or intravenous phosphate, and elevated DPG by as 
much as $10-20 \%$ in two subjects. We have used 30 mmoles of phosphate administered orally three times a day and obtained an increase in DPG levels over a five-day period of $15 \%$ in preadapting subjects to altitude (FIGURE 7)..$^{20}$ It has been possible in rhesus monkeys to increase DPG levels by as much as $50 \%$ with a combination of phosphate, inosine, and pyruvate. ${ }^{21}$ However, man lacks uricase, and a side effect of inosine administration in man is an elevation of uric acid levels.

It is imperative to investigate the significance of changes in oxygen affinity in order to validate further research into therapeutic manipulation of the oxygen dissociation curve. In rats, oxygen affinity appears to have little effect on exercise performance. However, exercising muscle extracts most of the oxygen from blood, and red cell oxygen affinity may have little to do with such extraction.

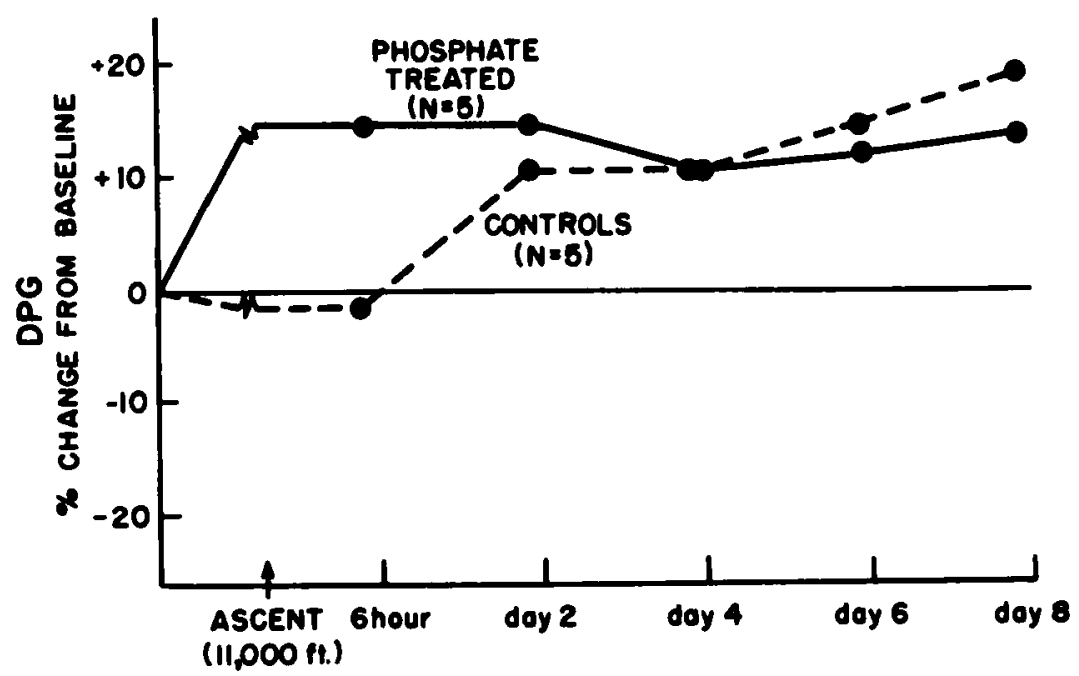

FIGURE 7. The effect of phosphate on red cell DPG levels in altitude preadaptation. Phosphate was given for $\mathbf{3 6}$ hours prior to ascent and for $\mathbf{7 2}$ hours after ascent. Reprinted by permission of Plenum Press.

On the other hand certain organs, such as the central nervous system and kidney, extract only about $25 \%$ of oxygen carried in the blood. That percentage should be significantly affected by red cell oxygen affinity, as discussed earlier. It is our belief that studies aimed at testing performance of organs such as the central nervous system, as affected by oxygen affinity during hypoxic stress such as altitude, are quite critical. So far nothing has been reported in this area, but it is an area of intensive investigation in our own laboratory.

\section{ACKNOWLEDGMENTS}

We wish to thank Academic Press for permission to reproduce Figure 1, which came from the second edition of The Red Blood Cell (Vol. I. 1974), 
D. M. Surgenor, Ed.; The Japanese Biochemical Society for permission to reproduce FIGURE 3, which came from J. Biochem. 59:139-144; Plenum Press for permission to reproduce FIGURE 5, which came from Hemoglobin and Red Cell Structure and Function (1972), G. J. Brewer, Ed.; and Plenum Press also for permission to reproduce FIGURE 7, which came from Oxygen Transport to Tissue (1973), H. Bicher and D. Burley, Eds.

\section{REFERENCES}

1. Brewer, G. J. 1974. The Red Blood Cell. 2nd edit. D. M. Surgenor, Ed. Chapter 11. Academic Press. New York, N.Y. In press.

2. Minakami, S. \& H. Yoshixawa. 1966. J. Biochem. (Tokyo) 59: 139.

3. RAPOPORT, S. 1968. In Essays in Biochemistry. P. N. Campbell \& G. D. Greville, Eds. Vol. 4: 69-103. Academic Press. New York, N.Y.

4. Rapoport, S. 1970. Bull. Soc. Chim. Biol. 52: 1169.

5. Yoshikawa, H. \& S. Minakami. 1968. Folia Haemat. 89: 357.

6. Minakami, S. \& H. Yoshikawa. 1966. J. Biochem. 59: 145.

7. Zürcher, C., J. Loos \& H. Prins. 1965. In Proc. 10th Congr. Intern. Soc. Blood Transfusion. S. Karger, Ed. : 549-556. Stockholm, Sweden.

8. Brewer, G. J. 1965. Biochem. Biophys. Res. Comm. 18: 430-434.

9. Oelshlegel, F. J., JR., G. J. Brewer, B. A. J. Sander \& N. A. Agar. 1973. Abstracted in Am. J. Hum. Genet. 25: 56A.

10. Brewer, G. J., J. Eaton, J. Weil \& R. Grover. 1970. In Red Cell Metabolism and Function : 95-114. G. J. Brewer, Ed. Plenum Press. New York, N.Y.

11. Oelshlegel, F. J., JR., G. J. Brewer, P. A. Penner \& E. B. Schoomaker. 1972. In Hemoglobin and Red Cell Structure and Function : 377-396. G. J. Brewer, Ed. Plenum Press. New York, N.Y.

12. Moore, L. G., G. J. Brewer \& F. J. Oelshlegel, JR. 1972. In Hemoglobin and Red Cell Structure and Function : 397-413. G. J. Brewer, Ed. Plenum Press. New York, N.Y.

13. Moore, L. G. 1973. Thesis for the Ph.D. degree. University of Michigan.

14. Moore, L. G., G. J. BreWer \& C. S. SiNG. Manuscript in preparation.

15. Oelshlegel, F. J., JR., G. J. Brewer \& C. S. Sing. Manuscript in preparation.

16. Brewer, G. J. 1967. Biochem. Genet. 1: 25-34.

17. Noble, N. A. \& G. J. Brewer. 1972. In Hemoglobin and Red Cell Structure and Function : 155-164. G. J. Brewer, Ed. Plenum Press. New York, N.Y.

18. NoBle, N. A. Unpublished observations.

19. BraIN, M. C. \& R. T. CARD. 1972. In Hemoglobin and Red Cell Structure and Function : 145-154. G. J. Brewer, Ed. Plenum Press. New York, N.Y.

20. Moore, L. G., G. J. Brewer, F. J. Oelshlegel, JR, \& A. M. Rose. 1974. In Oxygen Transport to Tissue : 693-698. H. Richer \& D. Braley, Eds. Plenum Press. New York, N.Y.

21. Sugerman, H. J., T. W. Pollock, E. F. Rosato, M. Delivoria-Papadopoulos, L. D. Miller \& F. A. OSKI. 1972. Blood 39: 525. 